\title{
Diagnosis and treatment of hemophagocytic lymphohistiocytosis in an adult patient with ehrlichiosis
}

\author{
J oel Provenzano' ${ }^{1}$ Timothy Kamerzell ${ }^{2}$, Eyad Reda², Dana Hawkinson ${ }^{2}$, Matthew Sharpe ${ }^{1}$ \\ 1. Department of Pulmonary and Critical Care Medicine, Kansas University Medical Center, Kansas, United States. \\ 2. Department of Medicine, Kansas University Medical Center, Kansas, United States.
}

Correspondence: Joel Provenzano. Address: Department of Pulmonary and Critical Care Medicine, Kansas University Medical Center, Kansas, United States. Email: jprovenzano@kumc.edu

Received: February 10, 2015

Accepted: March 2, 2015

Online Published: March 19, 2015

DOI : $10.5430 /$ crim.v2n2p44

URL: http://dx.doi.org/10.5430/crim.v2n2p44

\begin{abstract}
Hemophagocytic lymphohistiocytosis (HLH) is a rare and often fatal disease, characterized by uninhibited monocyte activation and tissue destruction from an immense inflammatory response. Most cases have been described in the pediatric population, though a rising number of cases have been identified in adults. Adult cases are typically secondary to a triggering process, of which infectious diseases such as Ehrlichia have been implicated. Our patient was an adult male who presented with multi-system organ failure secondary to HLH in the setting of documented Ehrlichia infection, with probable Epstein-Barr Virus (EBV) reactivation. His critical illness resolved after starting appropriate HLH therapy and specific treatment directed at Ehrlichia. This case demonstrates the importance of early recognition and treatment of HLH and its associated triggers in adult patients.
\end{abstract}

\section{Keywords}

Ehrlichia, Hemophagocytic lymphohistiocytosis, Ferritin, Sepsis, Critical care

\section{I ntroduction}

Hemophagocytic lymphohistiocytosis (HLH) is a rare and often fatal disease. Most cases have been described in the pediatric population, though a rising number of cases have been identified in adults. Adult cases are typically secondary to a triggering process, of which infectious diseases have been implicated. This case demonstrates the importance of early recognition and treatment of HLH and its associated triggers in adult patients.

\section{Case presentation}

\subsection{History}

A 47 year old Micronesian male was transferred to our institution from a rural hospital with altered mental status, progressive generalized weakness, tachycardia, tachypnea, and fever $\left(39^{\circ} \mathrm{C}\right)$. Due to his altered mental status, we were unable to obtain a detailed past history or family history. By report, the patient was not on any prior medications, and a 
cousin stated the patient was in good health one week prior to returning from a trip with his friends where significant alcohol consumption was thought to be involved. The patient worked in a food processing facility, but adverse work exposures were unclear. On physical exam, the patient was oriented to person only and responded with either yes or no to all questions asked. Also present was bilateral conjunctival injection, abdominal tenderness and guarding, an erythematous rash with blanching areas of skin over the upper chest, and 2+ bilateral lower extremity pitting edema. His review of systems was negative for complaints of chest pain, dyspnea, headache, neck pain, or photophobia.

\subsection{Hospital course}

The initial workup revealed multi-system organ failure with transaminitis, acute renal failure (creatinine 11.0mg/dL, with unknown baseline), leukopenia (3100/uL), and thrombocytopenia (22,000/uL). A coagulation panel was significant for an INR of 1.1, aPTT 73.2, and fibrinogen of 155. An extensive workup for toxic substances ingestion was negative. Chest $\mathrm{x}$-ray on admission did not reveal any acute process, and a magnetic resonance image (MRI) of the brain on hospital day 1 revealed non-specific white matter disease.

The patient was started on broad-spectrum antimicrobials for severe sepsis, including acyclovir for possible viral meningitis and doxycycline to cover for tick-borne associated illness. Hemodialysis was initiated, and a lumbar puncture was performed. Cerebrospinal fluid (CSF) analysis returned a total protein level of $137 \mathrm{mg} / \mathrm{dL}$ in the setting of a hemorrhagic tap (RBC 5,000,000 cells/uL), with a significantly elevated neutrophilic leukocytosis (2300 cells/uL, 96\% neutrophils). His mental status continued to deteriorate and he developed oral mucosal bleeding from his thrombocytopenia and coagulopathy, requiring intubation for airway protection.

With initial blood and CSF cultures negative, acyclovir was stopped and a Hematology consult was obtained. Concern was expressed for HLH, and a second battery of labs was sent. The results returned with a ferritin $>15,000 \mathrm{ng} / \mathrm{mL}$ (max of instrument) and triglycerides $1234 \mathrm{mg} / \mathrm{dL}$. A bone marrow biopsy was performed, revealing increased megakaryocytes and hemophagocytosis, as seen in Figures 1 and 2.

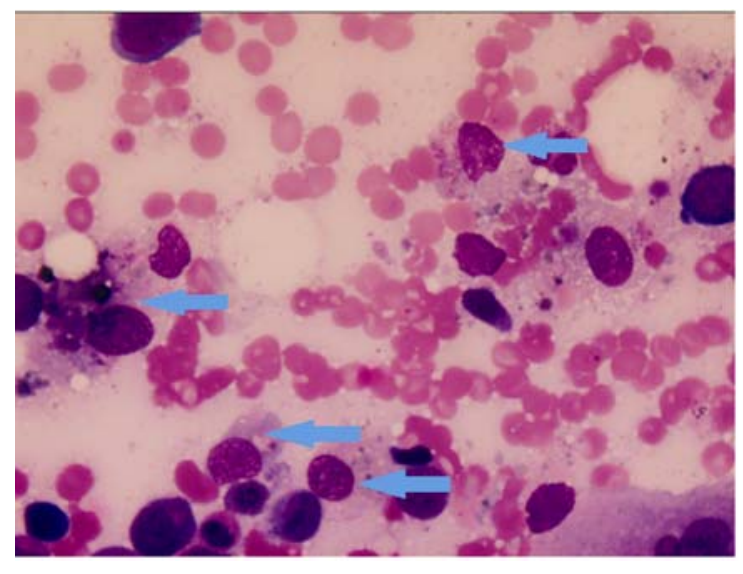

Figure 1. Bone marrow biopsy. Increased histiocytic activity; 4 histiocytes per high-power field. The large histiocyte to the left is filled with cellular debris.

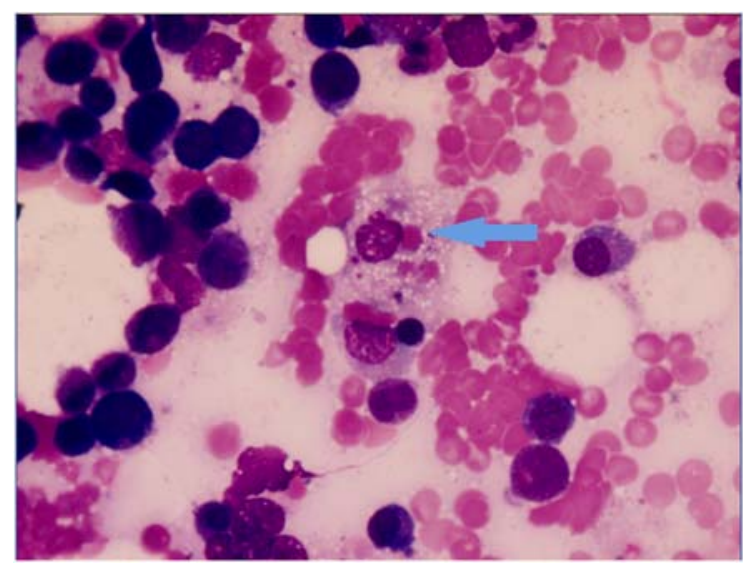

Figure 2. Bone marrow biopsy. Histocytes engulfing three red blood cells.

A blood sample sent for soluble IL-2 receptor levels later returned as $5900 \mathrm{pg} / \mathrm{mL}$ (normal $<1033 \mathrm{pg} / \mathrm{mL}$ ). Flow cytometry, bone marrow cytology, and $\mathrm{T}$ and $\mathrm{B}$ cell gene rearrangement studies excluded a contributing lymphomatous process.

A comprehensive evaluation for infectious causes was performed, and was negative for human immunodeficiency virus (HIV), hepatitis, cytomegalovirus (CMV), syphilis, herpes simplex virus, Brucella, Rocky Mountain Spotted Fever, and 
Lyme disease. A viral encephalitis panel and parasite screen was also negative. The infectious workup was positive for an EBV PCR of 5200 copies and 3100 copies in the serum and CSF respectively. EBV capsid IgG and EBV nuclear Ag antibodies were positive, but capsid IgM and early antigen antibodies were negative. Ehrlichia IgG titers were also positive at 1: 256, and both serum and CSF PCR were positive for Ehrlichia chaffeensis.

\subsection{Treatment plan}

Having met 6 out of 8 criteria for HLH [fever, hypertriglyceridemia (> $265 \mathrm{mg} / \mathrm{dL}$ ), hyperferritinemia (> $500 \mathrm{ng} / \mathrm{mL}$ ), elevated IL-2 receptors, cytopenia, and hemophagocytosis], treatment was directed at suppressing the patient's dysregulated immune response and attacking the infective triggers of the patient's disease. Doxycycline, started at admission, was also continued for a total of ten days at a dose of $100 \mathrm{mg}$ IV twice daily. Though his EBV viremia was likely reactivation, the patient completed four doses of rituximab ( $375 \mathrm{mg} / \mathrm{m}^{2} \mathrm{IV}$ ). He was immunosuppressed with dexamethasone (20 mg/m² IV) and etoposide (75 mg/m² IV-50\% reduced due to renal and hepatic dysfunction) as recommended by the HLH-94 protocol. Etoposide was initially planned for twice weekly dosing for two weeks then weekly to complete an eight week course; however, due to continued pancytopenia the dose intervals were changed to weekly from the start. Dexamethasone dosing was also decreased to a $2 \mathrm{mg}$ daily maintenance dose, with a slow taper planned as an outpatient.

\subsection{Response to treatment}

Within 36 hours of admission, mild clinical improvement was noted, likely due to initiation of doxycycline at admission. The patient's altered mental status completely resolved and he was extubated 72 hours after directed HLH treatment began. Improvement of his laboratory findings coincided with his clinical improvement. By the fourth day of treatment the patient was no longer platelet transfusion dependent, his ferritin level had dropped to $3777 \mathrm{ng} / \mathrm{mL}$, as shown in Figure 3 , and his acute liver failure had resolved.

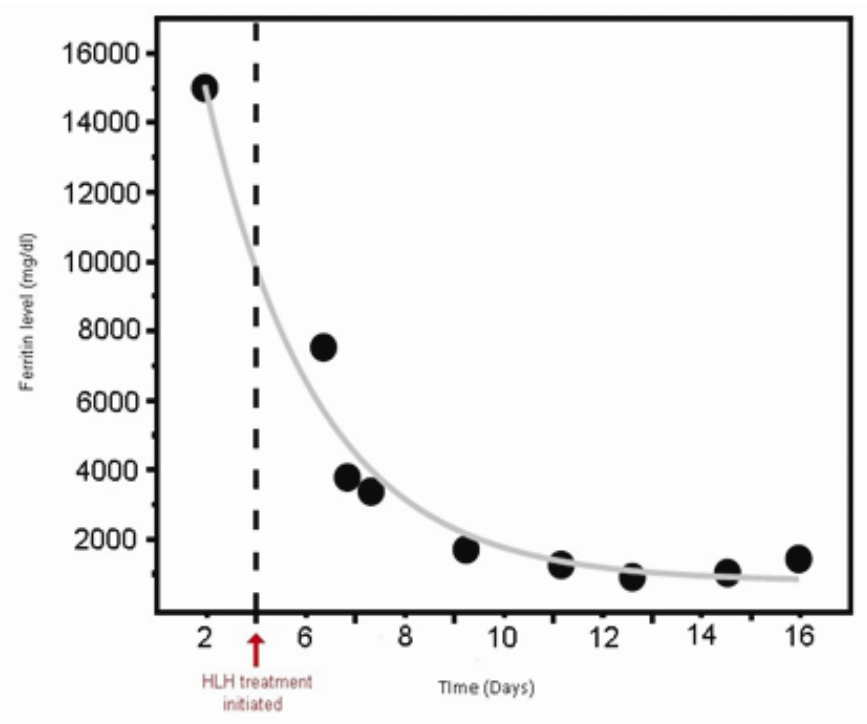

Ferritin serum levels response to HLH treatment

Figure 3. Ferritin levels

He completed his last dialysis treatment on post-treatment initiation day eleven, with a return of his creatinine to normal levels. The patient was discharged home on treatment day 16, with serum EBV PCR levels negative on that day. Repeat Ehrlichia titers were not drawn. He will finish his treatment course as an outpatient. 


\section{Discussion}

First described in 1952, HLH is a rare disease that is most commonly described in children. The highest incidence is in the infant population, those less than 3 months old ${ }^{[1]}$. Increasingly the disease is being identified in the adult population, with a review of 2197 adult cases revealing patients as old as 70 years old ${ }^{[1]}$.

Around $25 \%$ of cases are familial, with multiple genetic mutations identified. Patients with genetic mutations are more likely to have severe disease and present at an earlier age. UNC13D was the most common mutation in the white population (47\%), with PRF1 being most common in Hispanics (71\%), blacks (98\%), Arabs (36\%), and the Japanese ${ }^{[2]}$. The incidence has been increasing in children, most likely due to better recognition of the disease. It is possible that the disease has been under recognized in the adult population as well.

\subsection{I mportance of recognition}

Identifying patients with HLH early in their disease course is essential. Patients with a genetic mutation survive approximately 2 months without treatment, whereas patients treated with appropriate therapy have a median survival of $54 \%$ at 6.2 years ${ }^{[3]}$. However, the symptoms of HLH are similar to many other common conditions.

Patients with HLH tend to present with fever (96\%) ${ }^{[1]}$, hepatomegaly (95\%), cytopenias (> 80\%), elevated ferritin levels (25\%-93\%), elevated LDH (85\%), and elevated triglycerides (68\%) ${ }^{[3]}$. Patients may also have neurologic involvement, with abnormalities ranging from lethargy and confusion to seizures, encephalitis, and ataxia ${ }^{[2]}$. Later in the course of disease, and with more severe disease, patients might also develop renal dysfunction (16\%), rashes (25\%), or an ARDS-like syndrome $(42 \%)^{[1]}$.

The clinical findings of HLH are secondary to an immense inflammatory state, brought about by uninhibited macrophages and impaired cytotoxic cells ${ }^{[4]}$. Macrophages are antigen-presenting cells which secrete cytokines to attract cytotoxic cells, or T-lymphocytes that eliminate cells bearing antigen foreign to the host. In HLH this cytotoxic function is impaired, failing to destroy foreign cells or inhibit antigen-presenting macrophages. This triggers an unremitting cytokine cascade, which can cause excessive tissue damage ${ }^{[4]}$.

There are two main types of triggers that can lead to this impaired immunoresponse, those that lead to excessive immune activation, and those that stem from immunocompromise. Infection is the most common cause of immune activation triggers, with viral infections being the frequent culprit. Epstein-Barr virus (EBV) is the leading viral trigger, though cytomegalovirus, herpes simplex, parvovirus, varicella, and H1N1 influenza have been implicated as well ${ }^{[4]}$. Bacterial illnesses are less likely to cause HLH-type immune activation, but those with genetic immune hyper-responsive disorders like Kawasaki disease and X-linked lymphoproliferative disease are at high risk ${ }^{[2]}$. Congenital immunodeficiency leads the second category of HLH triggers, which also includes malignancy, HIV, and rheumatologic disease.

As stated above, without treatment the survival of patients with HLH is very poor. Some patients without severe disease and an identifiable trigger are able to go without treatment as the HLH syndrome will resolve with treatment of their triggering cause. However, this response is rare and only in the case of easily treatable infections. Most patients have a progressive course unless treatment and trigger are resolved. Despite treatment, many patients will still have a poor prognosis. These patients tended to have more neurological involvement ${ }^{[3]}$, age $<6$ months ${ }^{[3]}$, familial disease ${ }^{[3]}$, and higher serum ferritin levels at diagnosis ${ }^{[5]}$. Of survivors, lasting effects of the illness most commonly involved neurological deficits (19\%), from learning disabilities to severe mental retardation, along with other end-organ deficits ${ }^{[3]}$.

\subsection{Ehrlichiosis}

Our patient was also found to have positive Ehrlichia titers, a rare but previously reported trigger of HLH. Ehrlichia is transmitted by the Lone Star tick, which is carried by white-tailed deer, and infects monocytes, one of the cell types implicated in HLH. Two reports have described three different cases of HLH induced by Ehrlichia chaffeensis in children, 
each of which had full recovery after treatment with doxycycline +/- treatment with the HLH-94 or HLH-2004 protocol $^{[11,12]}$. One previous adult case has been reported, of a 67 year old male from New Mexico, who developed HLH secondary to infection with Ehrlichia canis ${ }^{[13]}$.

We hypothesize that Ehrlichiosis was the trigger of his HLH and that his EBV viremia was a secondary reactivation. It is unclear that he received benefit from rituximab in this case. He did show some mild clinical improvement prior to HLH-directed therapy, likely due to initiation of doxycycline at admission. However, with the severity of his immune dysregulation, it is certain he would not have recovered without the immunosuppressive regimen.

\subsection{HLH-94 Protocol}

Developed by the Histiocyte Society in 1994, the HLH-94 protocol is the current standard of treatment for HLH ${ }^{[3]}$. Changes to the treatment protocol were proposed in 2004, but the results of these changes have yet to be reported.

The diagnostic criteria for HLH, as shown in Table 1, however, are determined by the HLH-2004 protocol, and the criteria are the same regardless of patient age ${ }^{[2]}$.

Table 1. HLH-2004 Diagnostic Criteria

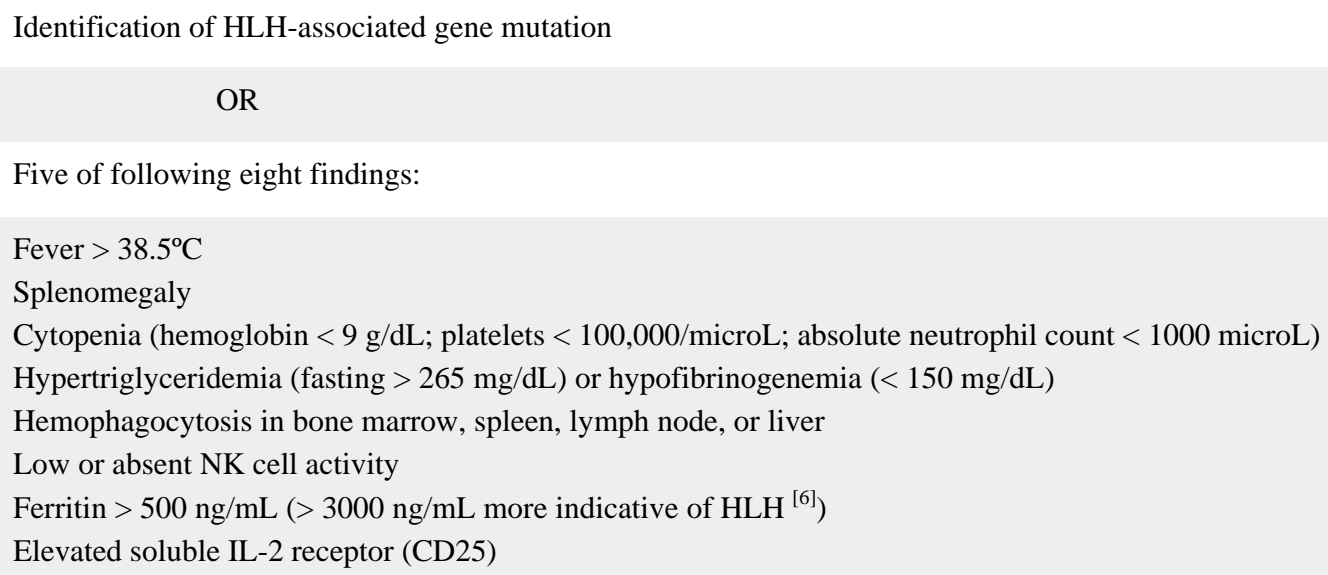

It is rare for a patient, as in our case, to meet all diagnostic criteria. While waiting for genetic testing to return, patients may often only exhibit three or four of the clinical criteria. As ferritin has been found to be such a specific marker for HLH when $>3000 \mathrm{ng} / \mathrm{mL}^{[6]}$, it has been suggested that patients with clinical findings suggestive of HLH and such an elevated ferritin should also be treated as having HLH.

The goal of the HLH-94 protocol is attempting to hamper the inflammatory cascade by suppressing the immune system. As previously stated, patients with mild or stable disease may respond simply with treatment of their triggering condition. However, most patients present with severe or progressive disease, requiring more significant intervention. The HLH-94 regimen is eight weeks of combined etoposide (VP-16) and high dose dexamethasone. Intrathecal methotrexate is also given for patients with central nervous system involvement ${ }^{\text {[7] }}$. Etoposide (VP-16) is administered twice weekly for two weeks, then weekly to complete the eight week regimen. The recommended dose for adults $150 \mathrm{mg} / \mathrm{m}^{2}$, with dose reductions for patients with renal impairment ${ }^{[2]}$. Dexamethasone is given in a tapering dose over the eight week course, initiating at $10 \mathrm{mg} / \mathrm{m}^{2}$ weekly for the first two weeks, then reducing the dose by half every two weeks until the course is completed.

Cyclosporine was initially a part of the HLH-94 protocol, with treatment beginning after the eight week course of etoposide (VP-16) and dexamethasone was completed. However, cyclosporine use was found to be associated with an increase in the incidence of posterior reversible encephalopathy syndrome (PRES) ${ }^{[3]}$, and is no longer recommended. 
Intrathecal methotrexate is advised in patients with HLH and confirmed central nervous system involvement. In infants the dose is based on age, but in all patients $>3$ years of age it is recommended to give $12 \mathrm{mg}$ in weekly doses until at least one week after central nervous system involvement has been eradicated ${ }^{3}$.

Supportive care is also a mainstay of treatment, with focus on preventing adverse effects of the disease or treatment. With severe cytopenia as a part of the disease process, replacement of blood products is the key, and transfusions should occur as indicated in other pancytopenic patients ${ }^{[7]}$. Infection is of concern, as many patients are already immunocompromised or have infection as an HLH trigger, and will be further immunosuppressed with HLH treatment. It is important to be mindful of opportunistic infections, and recommended to treat those with underlying immunodeficiency with intravenous immune globulin (IVIG) at a dose of $500 \mathrm{mg} / \mathrm{kg}^{[7]}$. Tight blood pressure control is the key as well, due to the risk of PRES as stated above.

The response to treatment is based mainly on the overall clinical improvement of the patient, however several diseasespecific recommendations are made. Ferritin levels should be monitored daily ${ }^{[2]}$ in the initial phase of treatment, as a corollary to inflammatory burden. For those with viral disease, serum viral titers should be monitored to ensure the triggering condition is being treated ${ }^{[2]}$. It is recommended to check soluble IL-2 receptor (CD25) levels weekly ${ }^{[2]}$, and those undergoing intrathecal treatments for CNS involvement should have CSF analysis with each weekly dose ${ }^{[2]}$.

Approximately half of patients treated with the HLH-94 protocol will be in remission one year after treatment has been completed ${ }^{[3]}$. If patients relapse in their disease, it is typically within the first year after their initial presentation ${ }^{[2]}$. Those that initially attained remission tend to do well with resumption of the same regimen as before ${ }^{[3]}$. If relapse happens during tapering of the initial induction course, it is recommended to restart the eight week regimen over at full doses. In cases of disease progression while on full dose treatment, Alemtuzumab (anti-CD52) has been shown to obtain partial treatment response in $86 \%$ of patients ${ }^{[8]}$.

As there is no resolution of an underlying trigger, relapse is more likely in patients with a genetic mutation. In fact, no patient in the HLH-94 study who had familial disease survived without undergoing hematopoietic cell transplant (HCT) ${ }^{[3]}$. HCT is also recommended for patients with CNS involvement and incurable hematologic malignancies ${ }^{[2]}$. Patients who will require HCT are recommended to complete the initial eight week induction course as per the HLH-94 protocol. It is then recommended to continue on etoposide $150 \mathrm{mg} / \mathrm{m}^{2}$ every two weeks, alternating with dexamethasone $10 \mathrm{mg} / \mathrm{m}^{2}$ for three consecutive days every two weeks, until HCT is able to be performed ${ }^{[2]}$. In those patients with lack of initial HLH-94 response and subsequent treatment with Alemtuzumab, $77 \%$ eventually were able to proceed with HCT $^{[8]}$. Patients who successfully underwent HCT had similar prognosis as patients without familial disease, with a five-year survival of $66 \%{ }^{[3]}$.

\subsection{Role of rituximab}

Patients who have continual exposure to a triggering condition will have difficulty resolving their HLH despite appropriate treatment. Though likely a secondary reactivation in our patient, as mentioned previously, EBV is the most common viral trigger for HLH. With impaired immune system function as a part of HLH pathophysiology, patients are often unable to combat infections on their own. Balamuth, et al. hypothesized that rituximab, an anti-CD20 monoclonal antibody, would have good effect in the treatment of EBV-related $\mathrm{HLH}^{[9]}$, as it had shown previous success in fulminant mononucleosis. They administered a single dose of rituximab $375 \mathrm{mg} / \mathrm{m}^{2}$ to their pediatric patient, who had a good response to treatment with minimal side effects ${ }^{[9]}$.

The largest case study reviewing treatment of EBV-related HLH with rituximab was completed by Challapandian, et al., in $2013^{[10]}$. They reviewed 42 patients with EBV-HLH, with a median serum viral load of 114,200 copies. Patients received 1-10 doses of rituximab, with a median of 3 doses at an average of $375 \mathrm{mg} / \mathrm{m}^{2}$ each dose. Patients had their initial dose on day 5.5 on average, range days $1-28$, and 9 patients received their doses for recurrent HLH. All patients received a 
combination of etoposide, dexamethasone, and/or cyclosporine as per HLH-94 protocol. 18 of their 42 patients (43\%) had clinical improvement, with an average 500-fold reduction in serum EBV viral load and 3.7-fold reduction in serum ferritin levels. 26 of their 42 patients were alive at the time of analysis, with a median survival of 1120 days, range 230-3750 days. The 16 deceased patients had all succumbed to HLH-related multi-organ system failure at a median of 97.5 days, range 22-900 days ${ }^{[10]}$. Rituximab is now recommended for treatment of HLH when $>10,000$ copies of EBV/mcg cellular DNA are detected in serum or CSF, with doses of $375 \mathrm{mg} / \mathrm{m}^{2}$ weekly for 1-4 weeks, contingent on how quickly the viral load $\operatorname{drops}^{[2]}$.

\section{Conclusion}

Hemophagocytic lymphohistiocytosis is a rare but very fatal disease, especially when untreated. Though previously thought to be a pediatric disease, the growing number of cases in the adult population is likely due to better recognition of this unique disease process. This case demonstrates the importance of understanding HLH as a potential cause of serious disease and identifying any and all possible triggers.

\section{References}

[1] Ramos-Casals M, Brito-Zerón P, López-Guillermo A, et al. Adult haemophagocytic syndrome. Lancet. 2014; 383: 1503. http://dx.doi.org/10.1016/S0140-6736(13)61048-X

[2] Jordan MB, Allen CE, Weitzman S, et al. How I treat hemophagocytic lymphohistiocytosis. Blood. 2011; 118: 4041. http://dx.doi.org/10.1182/blood-2011-03-278127

[3] Trottestam H, Horne A, Aricò M, et al. Chemoimmunotherapy for hemophagocytic lymphohistiocytosis: long-term results of the HLH-94 treatment protocol. Blood. 2011; 118: 4577. http://dx.doi.org/10.1182/blood-2011-06-356261

[4] Filipovich A, McClain K, Grom A. Histiocytic disorders: recent insights into pathophysiology and practical guidelines. Biol Blood Marrow Transplant. 2010; 16: S82. http://dx.doi.org/10.1016/j.bbmt.2009.11.014

[5] Lin TF, Ferlic-Stark LL, Allen CE, et al. Rate of decline of ferritin in patients with hemophagocytic lymphohistocytosis. Mayo Clin Proc. 2014; 89: 484. http://dx.doi.org/10.1002/pbc.22774

[6] Allen CE, Yu X, Kozinetz CA, et al. Highly elevated ferritin levels and the diagnosis of hemophagocytic lymphohistiocytosis. Pediatr Blood Cancer. 2008; 50: 1227. http://dx.doi.org/10.1002/pbc.21423

[7] Henter JI, Aricò M, Egeler RM, et al. HLH-94: a treatment protocol for hemophagocytic lymphohistiocytosis. HLH study Group of the Histocyte Society. Med Pediatr Oncol. 1997; 28: 342.

http://dx.doi.org/10.1002/(SICI)1096-911X(199705)28:5<342::AID-MPO3>3.0.CO;2-H

[8] Marsh RA, Allen CE, McClain KL, et al. Salvage therapy of refractory hemophagocytic lymphohistiocytosis with alemtuzumab. Pediatr Blood Cancer. 2013; 60: 101. http://dx.doi.org/10.1002/pbc.24188

[9] Balamuth NJ, Nichols KE, Paessler M, et al. Use of rituximab in conjunction with immunosuppressive chemotherapy as a novel therapy for epstein barr virus-associated hemophagocytic lymphohistiocytosis. J Pediatr Hematol Oncol. 2007; 29(8): 569. http://dx.doi.org/10.1097/MPH.0b013e3180f61be3

[10] Chellapandian D, Das R, Zelley K, et al. Treatment of epstein barr virus-induced haemophagocytic lymphohistiocytosis with rituximab-containing chemo-immunotherapeutic regimens. Br J Haematol. 2013; 162(3): 376. http://dx.doi.org/10.1111/bjh.12386

[11] Hanson D, Walter AW, Powell J. Ehrlichia-induced hemophagocytic lymphohistiocytosis in two children. Pediatr Blood Cancer. 2011; 56: 661. http://dx.doi.org/10.1002/pbc.22814

[12] Burns S, Saylors R, Mian A. Hemophagocytic lymphohistiocytosis secondary to ehrlichia chaffeensis infection: a case report. J Pediatr Hematol Oncol. 2010; 32(4): e142. http://dx.doi.org/10.1097/MPH.0b013e3181c80ab9

[13] Abbott KC, Vukelja SJ, Smith CE, et al. Hemophagocytic syndrome; a cause of pancytopenia in human ehrlichiosis. Am J Hematol. 2006; 38: 230. http://dx.doi.org/10.1002/ajh.2830380315 